\title{
Development of Omni-Directional Proximity Sensor for Drone
}

\author{
Satoshi Tsuji ${ }^{\mathrm{a}, *}$, Teruhiko Kohama ${ }^{\mathrm{a}}$ \\ aFukuoka University, 8-19-1 Nanakuma, Jonan-ku, Fukuoka 814-0180, Japan \\ *Corresponding Author: tsuji@fukuoka-u.ac.jp
}

\begin{abstract}
Unmanned aerial vehicles including drones are attracting attention in recent years. A drone is required to approach an object and obstacles when the drone uses for inspection and an indoor space. However, the collision risk is increased when the drone is close to the object. In this paper, we propose the omni-directional proximity sensor for a drone to avoid a collision. The sensor system consists of 30 units of time-of-flight (ToF) sensors that are able to detect the distance between the sensors and objects. The sensor system attaches to the propeller guard and the drone, and detects the object at non-contact area. Thus, this sensor system can avoid unnecessary contact between the drone and objects. Therefore, the sensor system may be useful for drones.
\end{abstract}

Keywords: proximity sensor, drone, collision avoidance.

\section{Introduction}

Unmanned aerial vehicles are attracting attention in recent years. In particular, drone is expected to be used in various fields such as photography, agriculture, delivery, inspection, and product management. Among these, we focused on the drone for inspection and product management. Many drone system have been proposed for inspection of transmission line ${ }^{(1)-(2)}$, concrete ${ }^{(3)}$ of a tunnel and bridge, and so on. The drone for product management in indoor space ${ }^{(4)}$ has been proposed. Many of these drones are required to approach an object and obstacles when the drone uses for inspection and an indoor space. However, the collision risk is increased when the drone is close to the object. The main methods to avoid collisions between the drone and obstacles are a manual control by the pilot and an automatic control using sensor information. In the case of manual control by the pilot, the collision avoidance depends on the pilot's skills. There is a risk of the collision by blind spots and maneuvering errors, even if the pilot has good skills.

On the other hand, many sensors for drone have been proposed to avoid collision ${ }^{(5)-(10)}$. The drone with a laser finder has been proposed ${ }^{(5)}$. A laser finder can detect an object with high accuracy in the horizontal direction. However, many laser scanners are heavy and expensive. The method of collision avoidance on quadrotor unmanned aerial vehicle using image sensors has been proposed ${ }^{(6)}$. However, image processing requires an advanced processing system. The drone with some ultrasonic sensors has been proposed (7)-(8). Ultrasonic sensors are one of the useful sensors for drone because they can detect the distance to the object and the size is not large. Furthermore, a system combining ultrasonic sensors and optical sensors on a drone has also been proposed ${ }^{(9)}$. However, many ultrasonic sensor are not small enough, and it is not easy to attach many sensor on a drone. Several time-of-flight (ToF) sensors are commercially available in recent years ${ }^{(10)}$. This ToF sensor is inexpensive, small in size, and can detect a distance. In addition, the small ToF sensors on a drone have been proposed ${ }^{(11)}$. However, the system consists of 4 pieces of ToF sensors, and many blind spots may be occurred.

An objective of this study is to establish a proximity sensor system without blind spots for a drone. Fig. 1 shows a goal image of the proposed sensor system on a drone. The

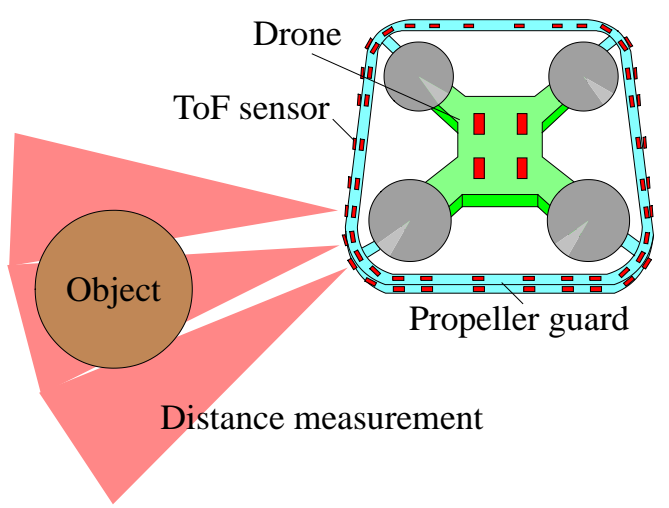

Fig. 1. Goal image of this study. 
sensor system increases safety of drone because the sensor constantly measures a state of near the drone, and uses to avoid contact with objects.

Previously, we have developed a proximity skin sensor using ToF sensors that can be attached to the entire human cooperative robots surface for safety ${ }^{(12)}$. The sensor comprises 54 pieces of ToF sensors that can detect the distance between the sensor and an object. The detection range of this sensor system is about $300 \mathrm{~mm}$. We think that it is useful as detection range for a robot, however it is short for a drone.

In this paper, we proposed an omni-directional proximity sensor for drone. This sensor system consists of 30 pieces of ToF sensors. ToF sensors are different from that of previous study (12) to increase the detection range. The detection range of this sensor system is about $2000 \mathrm{~mm}$. The prototype sensor is set on the propeller guard and top of the drone. The sensor measures the distance between the sensor and objects while the drone is flying.

\section{Proposed Sensor System}

\subsection{Omni-Directional Proximity Sensor}

The sensor system consists of many ToF sensors. ToF sensor detects the distance to an object by measuring the time to receive light reflected from the object when infrared light is emitted. Thus, a ToF sensor can measure the distance without affecting the reflectivity of the object. Fig. 2 show diagram of the prototype sensor. The prototype sensor used VL53L0X (STMicroelectronics, Inc.) ${ }^{(10)}$ as ToF sensor. The size of VL53L0X is about $5 \times 3 \mathrm{~mm}$. The sensor board (about $15 \times 10 \mathrm{~mm}$ ) including VL53L0X is used for easy prototyping. The size of the sensor system can be decreased by using VL53L0X instead of the sensor board in the future. VL53L0X can be set in the software setting with a detection distance of $1200 \mathrm{~mm}$ detection mode and $2000 \mathrm{~mm}$ detection mode. In this study, the $2000 \mathrm{~mm}$ detection mode was used. The prototype sensor consists of 5 units of the sensor module. The sensor module consists of 6 pieces of ToF sensors and a slave microcomputer. The VL53L0X is connected to a slave microcomputer by $\mathrm{I}^{2} \mathrm{C}$ serial bus 2 , and controlled by the slave microcomputer. Devices with different $\mathrm{I}^{2} \mathrm{C}$ addresses can be connected on one $\mathrm{I}^{2} \mathrm{C}$ serial bus. The $\mathrm{I}^{2} \mathrm{C}$ device address of VL53L0X can be changed to a new address using the I/O of the slave microcomputer and software. Each slave and master microcomputer is connected by $\mathrm{I}^{2} \mathrm{C}$ serial bus 1 . The $\mathrm{I}^{2} \mathrm{C}$ device address of each slave microcomputer is set to

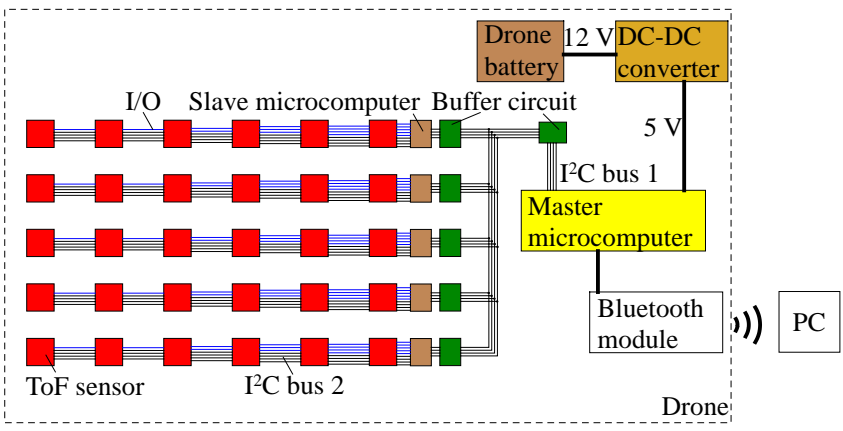

Fig. 2. Schematic diagram of the proposed sensor.

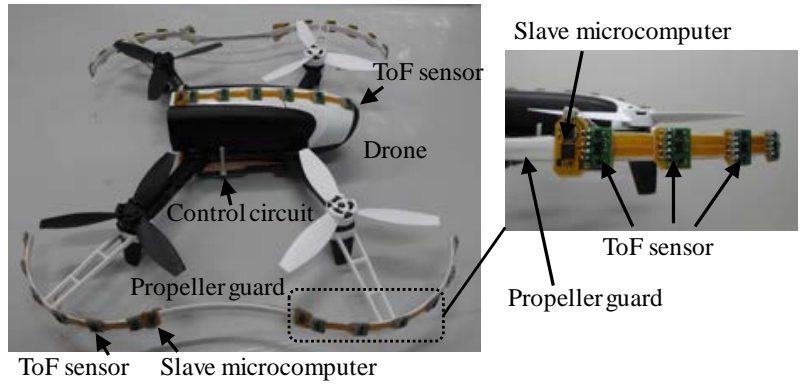

Fig. 3. Prototype sensor on the drone.

a different address. Thus, the sensor system saves wiring because the sensor modules are connected by one $\mathrm{I}^{2} \mathrm{C}$ bus 1 . Buffer circuits are used between the master and slave microcomputers to isolate the $\mathrm{I}^{2} \mathrm{C}$ bus capacitance ${ }^{(12)}$. Angle of divergent laser emission for the VL5310X is 25 degrees, and the laser output of VL53L0X is within Class 1 laser safety limit. The peak of IR emitting light of VL53L0X is $940 \mathrm{~nm}$. The interval between each ToF sensor is $30 \mathrm{~mm}$. Fig. 3 shows the prototype sensor on the drone (Parrot, Bebop Drone2). The sensor system is attached to the drone and the commercial propeller guard. 4 units of the sensor modules are attached to the propeller guard, and a unit of sensor module is attached to the top of the drone. Thus, the sensor system can detect objects in the horizontal and upside of the drone. The measured data are transmitted from the master microcomputer to the PC using a bluetooth module. The power supply ( $5 \mathrm{~V}$ ) of the sensor system is changed using a DC-DC converter from the drone battery (12 V). It takes approximately $38 \mathrm{~ms}$ to obtain one complete cycle of measurements (30 sensors) including a data transmitting time from the bluetooth module to PC.

\subsection{X-Y Position Detection}

We calculated the $\mathrm{X}-\mathrm{Y}$ position of the object using each ToF sensor. Fig. 4 shows the image of $X-Y$ position detection of the object using ToF sensors. The object position $\left(X_{n}, Y_{n}\right)$ 


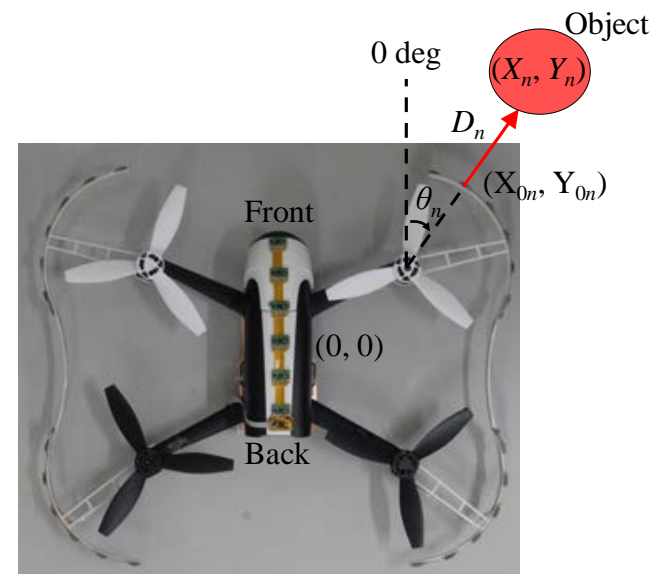

Fig. 4. X-Y position detection of the object using each ToF sensor.

is determined by the distance information $\left(D_{n}\right)$ from ToF sensor and the position $\left(X_{0 n}, Y_{0 n}\right)$ and angle $\left(\theta_{n}\right)$ of ToF sensor. The center position of the drone is position $(0,0)$, and the front of the drone is 0 degree. $\theta_{n}$ is determined around the center axis of each propeller because this propeller guard has curved face around the center axis of each propeller. $\left(X_{n}, Y_{n}\right)$ is calculated by the following equations.

$$
\begin{aligned}
& X_{n}=X_{0 n}+D_{n} \sin \theta_{n} \\
& Y_{n}=Y_{0 n}+D_{n} \cos \theta_{n}
\end{aligned}
$$

A state of near the drone can be obtained by calculating each $\left(X_{n}, Y_{n}\right)$ of 24 pieces of ToF sensors on the propeller guard.

\section{Results and Discussion}

\subsection{Basic Characteristics of Prototype Sensor}

As a basic experiment, the drone with the prototype sensor is placed on a table, as shown in Fig. 5, whereas the sensor measure the distance to the object. The distance between the object and the sensor is set from 0 to $2000 \mathrm{~mm}$. The objects are the white styrene foam and the brown corrugated board. The ambient light condition is the daylight white light emitting diode (LED) ceiling lighting of the room. Fig. 6 show the measurement distance $(D)$. The sensor can detect the distance because $D$ is changed according to the distance shown in Fig. 6. In the case of the brown corrugated board, the detected distance is approximately $1500 \mathrm{~mm}$. In the case of the while styrene foam, the detected distance is approximately $2000 \mathrm{~mm}$. Although the detectable distance differs depending on the color (reflectance) of the object, the sensor can detect the distance from the object. Thus, ToF sensors on the drone can detect non-contact condition around the drone.

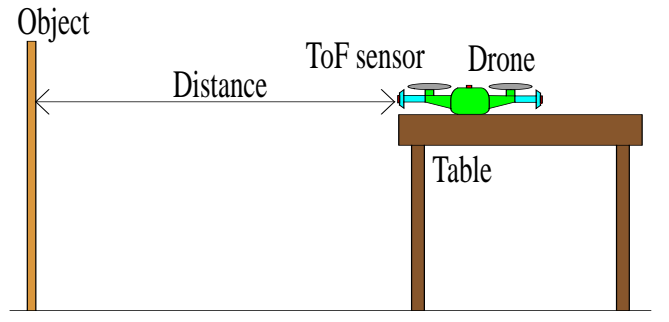

Fig. 5. Experimental conditions of the measurement distance on ToF sensor.

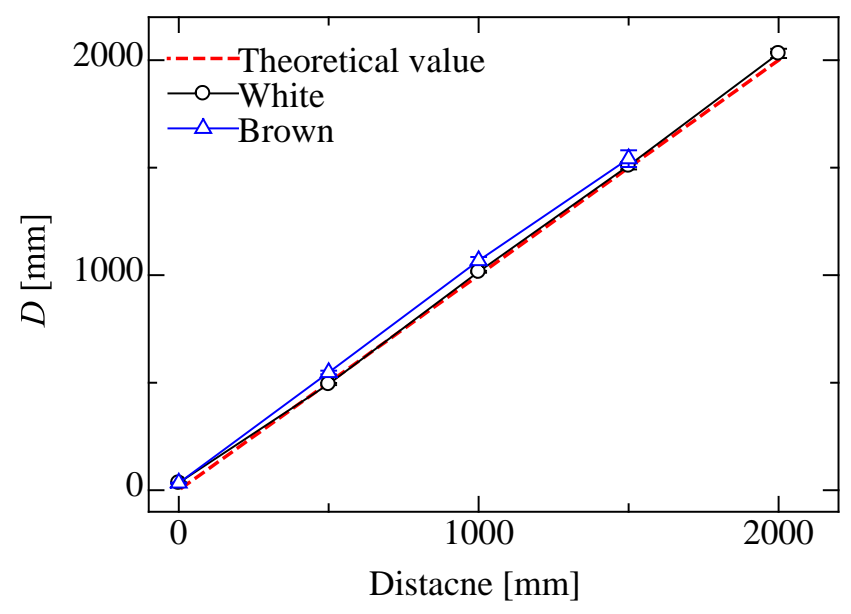

Fig. 6. The results of the measurement distance $(D)$.

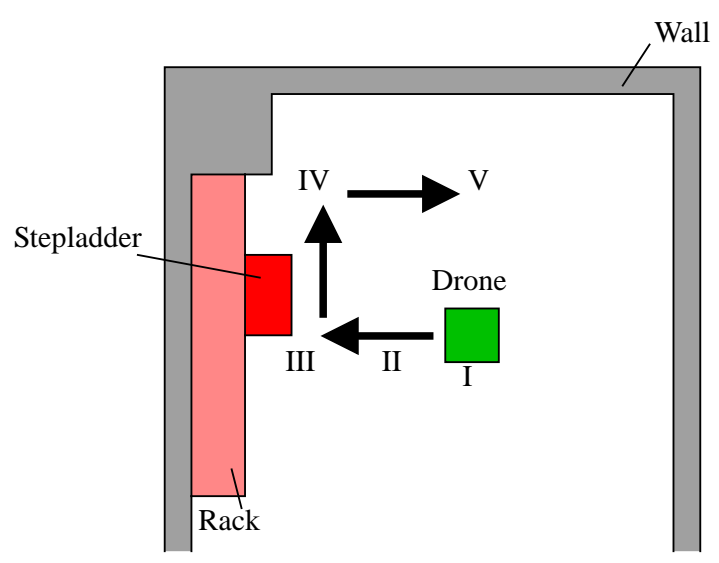

Fig. 7. Experimental conditions of flying drone.

\subsection{Object Detection on the Flying Drone}

We evaluated object detection on flying drone. Fig. 7 shows the experimental conditions. The drone flew indoors, and the objects are the rack and the stepladder. In this experiment, the drone flew at a fixed height. The drone moved along the arrow in Fig. 7, and Fig. 7(I)-(V) are the position of the drone. Fig. 8 shows the conditions of an experiment and results. The results of each ToF sensor are shown in the display in Fig. 8. The center point of the display shows the drone, and the red square is the detection $\mathrm{X}-\mathrm{Y}$ position of each ToF sensor. The $\mathrm{X}-\mathrm{Y}$ position was calculated 
(I)

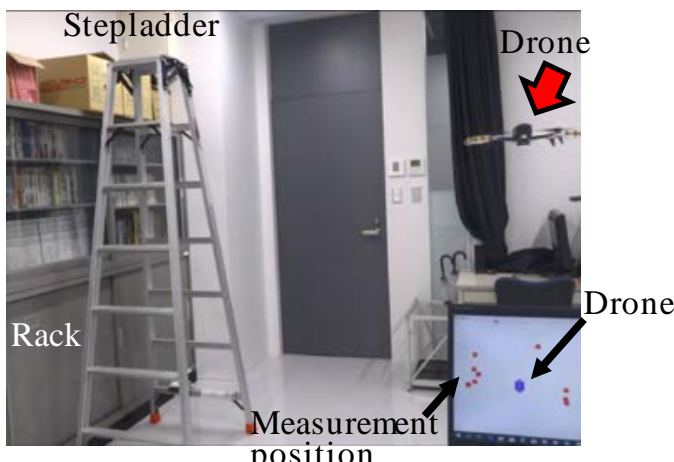

(II)

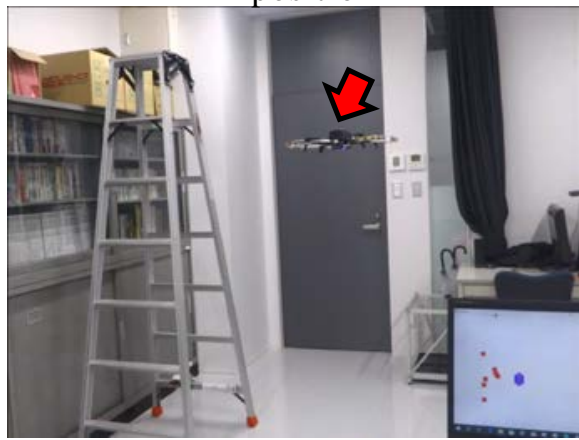

(III)

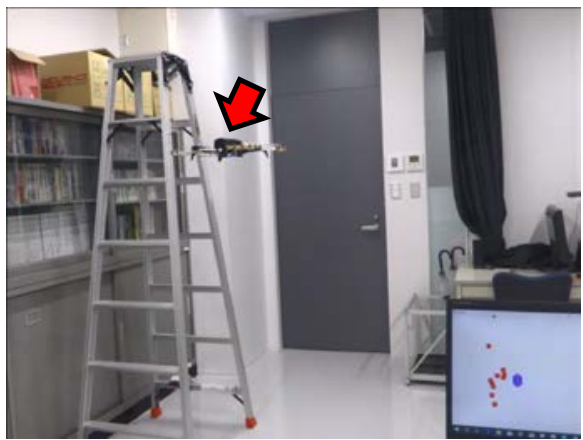

(VI)

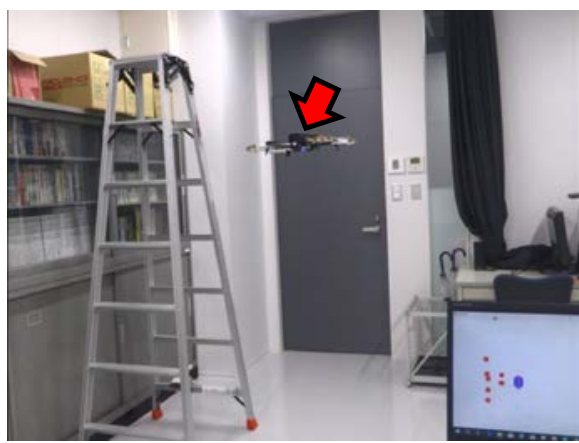

(V)

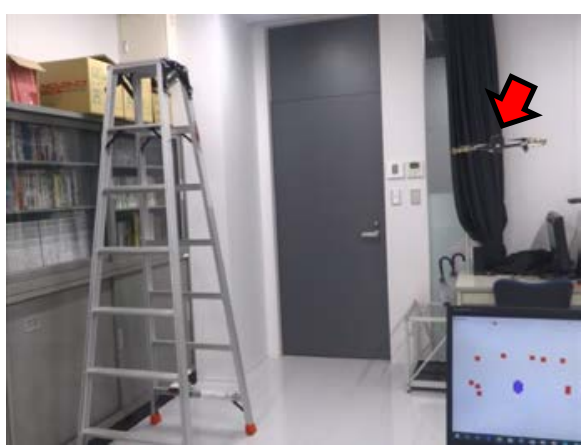

Fig. 8. The results of ToF sensors on the flying drone. by equations (1) and (2). The sensors in Fig. 8(I) and (V) detect the wall of the room. In addition, the distance between the ceiling and the drone can be detected. The sensor in Fig. 8(II)-(IV) detects the stepladder and rack. Thus, the sensor on the flying drone can detect the distance and shape of objects in real time. The detection distance of this ToF sensor is $2000 \mathrm{~mm}$, and it may be not enough for drone. However, the speed of the drone is usually slow during inspection and indoor. Thus, we think that the drone with the proposed sensor system can avoid unnecessary contact using the obtain information of the sensor. In this prototype sensor, the sensor system can detect objects in the horizontal and above the drone. We plan to attach many ToF sensors for multiple directions, and measure a state of near the drone without blind spots. From these results, we believe that the proposed proximity sensor can detect an object near the drone and can be used as a safety measure for drones.

\section{Conclusions}

We proposed an omni-directional proximity sensor for drone to avoid contact with objects. This sensor system consists of 30 pieces of ToF sensors. The ToF sensor can detect the distance from approximately $2000 \mathrm{~mm}$. The prototype sensor is set on the propeller guard and top of the drone. The sensor system on the flying drone can detect objects in the horizontal and above the drone. Thus, the drone can avoid making contact with the object using measured information. We believe that the proposed sensors can be used as proximity sensors for drones.

\section{Acknowledgment}

This work was supported in part by funds (No. 185009) from the Central Research Institute of Fukuoka University.

\section{References}

(1) Z. Li, Y. Liu, R. Hayward, J. Zhang and J. Cai : "Knowledge-based Power Line Detection for UAV Surveillance and Inspection Systems”, Proceedings of the 23rd International Conference on Image and Vision Computing New-Zealand (IVCNZ), 2008

(2) L. F. L. Vega, B. C. Toledo, A. Loukianov and L. E. G. Jimenez : "Power Line Inspection Via an Unmanned Aerial System Based on the Quadrotor Helicopter”, Proceedings of 17th IEEE Mediterranean Electrotechnical Conference, pp. 393-397, 2014 
(3) L. Yang, B. Li, W. Li, Z. Liu, G. Yang and J. Xiao : “A Robotic System Towards Concrete Structure Spalling And Crack Database, Proceedings of 2017 IEEE International Conference on Robotics and Biomimetics (ROBIO), pages 1276-1281 , 2017

(4) Y. Ma, N. Selby and F. Adib : "Drone Relays for BatteryFree Networks", Proceedings of the Conference of the ACM Special Interest Group on Data Communication, pp. 335-347, 2017

(5) F. Wang, J. Cui, S. K. Phang, B. M. Chen and T. H. Lee : "A Mono-Camera and Scanning Laser Range Finder Based UAV Indoor Navigation System”, Proceedings of 2013 International Conference on Unmanned Aircraft Systems, pp. 694-701, 2013

(6) M. K. Cheong, M. R. Bahiki and S. Azrad : "Development of collision avoidance system for useful UAV applications using image sensors with laser transmitter”, IOP Conference Series: Materials Science and Engineering, Vol. 152, p. 012026, 2016

(7) J. S. G. Guerrero, A. F. C. González, J. I. H. Vega and L. A. N. Tovar : "Instrumentation of an Array of Ultrasonic Sensors and Data Processing for Unmanned Aerial Vehicle (UAV) for Teaching the Application of the Kalman Filter”, Procedia Computer Science, Vol. 75, pp. 375 -380, 2015

(8) N. Gageik, T. Müller and S. Montenegro : "Obstacle Detection and Collision Avoidance Using Ultrasonic Distance Sensors for an Autonomous Quadrocopter", Proceedings of UAVveek Workshop Contrib., 2012

(9) N. Gageik, P. Benz and S. Montenegro : "Obstacle Detection and Collision Avoidance for a UAV With Complementary Low-Cost Sensors”, IEEE Access, Vol. 3, pp.599-609, 2015

(10) VL53L0X, https://www.st.com/resource/en/datasheet/ vl5310x.pdf, access date: May 25, 2019

(11)K. Nohara, S. Kawabata, J. H. Lee, S. Okamoto, H. Suzuki, T. Takiguchi and O. S. Park : "Development of Drone System Embedded with Multiple Distance Sensors for Inspection Task of Social Infrastructure”, Proceedings of IMECS 2018, 2018

(12) S. Tsuji and T. Kohama : "Proximity Skin Sensor using Time-of-flight Sensor for Human Collaborative Robot”, IEEE Sensors Journal, Vol. 19, No. 14, pp. 5859-5864, 2019 